\title{
Shaping of STDP curve by interneuron and $\mathrm{Ca}^{2+}$ dynamics Lynsey McCabe*1, Paolo Di Prodi ${ }^{1}$, Bernd Porr ${ }^{1}$ and Florentin Wörgötter ${ }^{2}$
}

Address: ${ }^{1}$ Department of Electronics and Electrical Engineering, University of Glasgow, Glasgow G12 8LT, UK and ${ }^{2}$ Bernstein Center for Computational Neuroscience, University of Göttingen, Bunsenstr. 10 (at the MPI), D-37073 Göttingen, Germany

Email: Lynsey McCabe* - lmc@elec.gla.ac.uk

* Corresponding author

from Sixteenth Annual Computational Neuroscience Meeting: CNS*2007

Toronto, Canada. 7-12 July 2007

Published: 6 July 2007

BMC Neuroscience 2007, 8(Suppl 2):P83 doi:I0.1 I86/I47I-2202-8-S2-P83

(c) 2007 McCabe et al; licensee BioMed Central Ltd.

Spike-timing-dependent-plasticity (STDP) $[1,2]$ is a special form of Hebbian learning [3] where the relative timing of post- and presynaptic activity determines the change in synaptic weight. More familiarly, the postsynaptic and presynaptic activity correspond respectively to the derivative of the membrane potential $\mathrm{V}_{\mathrm{m}}$ and the NMDA channel activation [4]. We present a model where the postsynaptic activity is modelled by the derivative of the $\mathrm{Ca}^{2+}$ concentration. Using a model of a pyramidal cell, attached interneuron and detailed $\mathrm{Ca}^{2+}$ dynamics, we show that the classical STDP curve is greatly altered, in particular, that long term depression (LTD) is markedly reduced [5] while LTP remains close to the original expected weight-change curve. In addition to this we have shown that by reducing the NMDA activity in the circuit model there is a noticeable change in the LTD/LTP magnitude in the STDP weight-change curve. This modification causes two effects; it reduces plasticity in the excitatory neuron but also reduces inhibition on the excitatory neuron. Therefore we show that by decreasing NMDA activity there is a clear reduction in LTD and LTP. This appears much like the "classical" STDP curve albeit scaled down in ratio to the reduced NMDA activity. In this study we have shown that the inhibitory interneuron reduces the LTD part of the STDP weight change curve. The more inhibition seen, the less LTD in the excitatory neuron. Thus, a hypofunction of inhibitory neurons will lead to more LTD in cortical structures and ultimately to less cortical activity. This hypofunction could be a possible mechanism of how administration of the NMDA antagonist PCP causes cortical hypoactivity[6] after a time lapse of a few days, and is already a topic of interest in the research of schizophrenia.

\section{References}

I. Magee JC, Johnston D: A synaptically controlled, associative signal for Hebbian plasticity in hippocampal neurons. Science 1997, 275:209-2I3.

2. Markram H, Lübke J, Frotscher M, Sakmann B: Regulation of synaptic efficacy by coincidence of postsynaptic Aps and EPSPs. Science 1997, 275:213-215.

3. Hebb DO: The organization of behaviour: A neuropsychological study. Wiley Interscience, New York; 1949.

4. Porr B, Saudargiene A, Wörgötter F: Analytical solution of spiketiming dependent plasticity based on synaptic biophysics. In Advances in neural information processing systems, 16, 2004 Cambridge MA: MIT Press.

5. Aihara $Y$, Abiru $Y$, Yamazaki $Y$, Wantanbe H, Fukushima $Y$, Tsukada $M$ : The relation between spike-timing dependent plasticity and $\mathrm{Ca}^{2+}$ dynamics in the hippocampal CAl network. Neuroscience 2007, 145:80-87.

6. Morris BJ, Cochran SM, Pratt JA: PCP: from pharmacology to modelling schizophrenia. Current Opinion in Pharmacology 2005, 5:101-106. 